An immersed boundary-based large-eddy simulation approach to predict the performance of vertical axis tidal turbines

Pablo Ouro Barba, Thorsten Stoesser

PII: S0045-7930(17)30120-2

DOI: 10.1016/j.compfluid.2017.04.003

Reference: $\quad$ CAF 3444

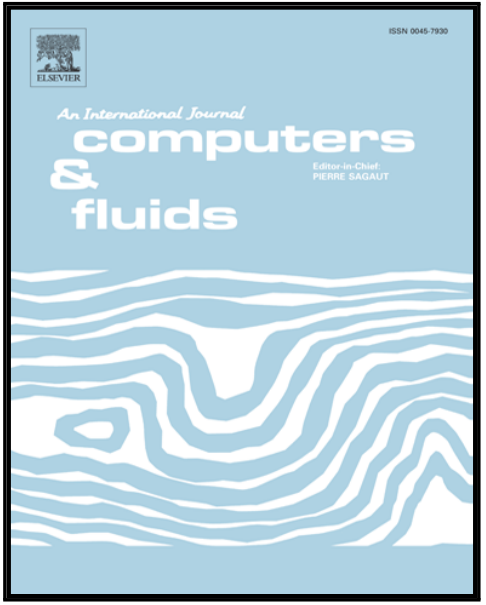

To appear in:

Computers and Fluids

Received date:

20 December 2016

Revised date:

23 February 2017

Accepted date:

6 April 2017

Please cite this article as: Pablo Ouro Barba, Thorsten Stoesser, An immersed boundary-based largeeddy simulation approach to predict the performance of vertical axis tidal turbines, Computers and Fluids (2017), doi: 10.1016/j.compfluid.2017.04.003

This is a PDF file of an unedited manuscript that has been accepted for publication. As a service to our customers we are providing this early version of the manuscript. The manuscript will undergo copyediting, typesetting, and review of the resulting proof before it is published in its final form. Please note that during the production process errors may be discovered which could affect the content, and all legal disclaimers that apply to the journal pertain. 


\section{Highlights}

- A LES solver is equipped with an IB method to predict the performance of VATTs.

- The method is validated with body-fitted models for a VATT in a laminar flow.

- Simulations of a VATT under turbulent flow confirmed the method's accuracy.

- LES-IB method results overcome RANS predictions with a closer match with experiments.

- Blade-vortex interaction visualisations highlight the complex flow around VATTs.

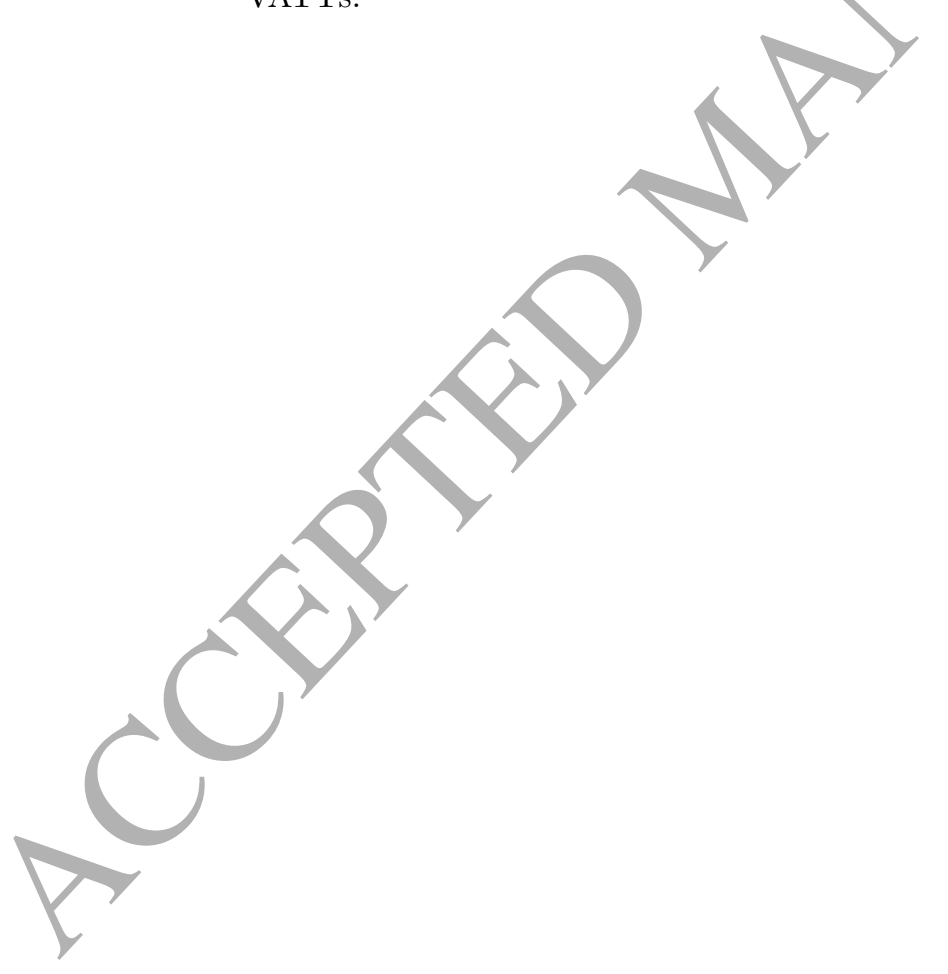




\title{
An immersed boundary-based large-eddy simulation approach to predict the performance of vertical axis tidal turbines
}

Pablo Ouro*, Thorsten Stoesser

Hydro-environmental Research Centre, Cardiff School of Engineering, Cardiff University. The Parade CF24 3AA, Cardiff, UK

\begin{abstract}
Vertical axis tidal turbines (VATTs) are perceived to be an attractive alterna-
\end{abstract} tive to their horizontal axis counterparts in tidal streams due to their omnidirectionality. The accurate prediction of VATTs demands a turbulence simulation approach that is able to predict accurately flow separation and vortex shedding and a numerical method that can cope with moving boundaries. Thus, in this study an immersed boundary-based large-eddy simulation (LES-IB) method is refined to allow accurate simulation of the blade vortex interaction of VATTs. The method is first introduced and validated for a VATT subjected to laminar flow. Comparisons with highly-accurate body-fitted numerical models results demonstrate the method's ability of reproducing accurately the performance and fluid mechanics of the chosen VATT. Then, the simulation of a VATT under turbulent flow is performed and comparisons with data from experiments and results from RANS-based models demonstrate the accuracy of the method. The vortex-blade interaction is visualised for various tip speed ratios and together with velocity spectra detailed insights into the fluid mechanics of VATTs are provided.

Keywords: Vertical Axis Turbines, Immersed Boundary method, Large-Eddy Simulation, Direct forcing, Tidal turbines, Vortex-blade interaction

\footnotetext{
*Corresponding author: OuroBarbaP@cardiff.ac.uk

Email addresses: OuroBarbaP@cardiff.ac.uk (Pablo Ouro), Stoesser@cardiff.ac.uk (Thorsten Stoesser)
} 


\section{Introduction}

The growing energy demand and the effects of carbon dioxide pollution from fossil-fuel burning amplify the need for technologies that can harness carbon free energy from natural resources. The interest in tidal stream energy has been

5 growing for the last years motivated mainly by two advantages (when compared to wind energy): (1) tidal streams are highly predictable and thus the energy that can be extracted from these streams through tidal turbines $[1,2]$ can be estimated until the end of their lifetime (say for the next 20-30 years); and (2) the density of water is approx. 800 times greater than the density of air and hence tidal turbines are relatively small in comparison to their wind turbine counterparts. An obvious disadvantage of tidal stream energy is the harsh and challenging environment in which the turbines are operating in being constantly subjected to strong intermittent fluid forces which calls into question their survivability or implies substantial operation and maintenance costs, 15 respectively [3].

Almost all tidal energy projects to date employ the horizontal axis tidal turbine (HATT) concept, probably by importing some of the technology from wind industry where horizontal axis turbines are dominating the (wind turbine) landscape. However, in a tidal stream environment vertical axis tidal turbines 20 (VATTs) offer several advantages: (1) they are omni-directional, i.e. their operation and efficiency is independent of the flow direction; (2) they rotate at lower Tip Speed Ratio (TSR) than HATTs, which is believed to be environmentally more friendly reducing noise generation [4] and fish mortality [5], and supposedly works better in low-to-medium tidal velocities as the cut in speed islower; and (3) they can make better use of shallow tidal stream flows as they maximise the use of the available cross-section. However, their efficiency is lower than horizontal axis turbines and self-starting could be problematic [6].

Most of the current knowledge on the performance of VATTs has been obtained from experimental work. Kiho et al. [7] tested a Darrieus-type turbine 

capacity changes. Roa et al. [8] analysed the performance of a VATT in a confined water tank with and without being ducted. The influence of the turbine's solidity was studied by McLaren [9] while Fiedler et al. [10] focused on turbine parameters such as blade and shaft shapes, pitch angle or the location of blade 35 attachment to the shaft. Recent studies looked at the effects of blade roughness [11] or turbine helicity [12] on the performance of VATTs. A complete review of recent tidal turbine technologies is provided in [6].

Experimental studies are costly and time-consuming and the avalable information from these tests is not enough to fully understand the complex fluid40 structure interaction of vertical axis tidal turbines, Numerical models, if accurate and trustworthy, can provide a more complete picture of the complex flow-turbine-interplay. However, highly turbulent flows and the complex fluidstructure interaction of a rotating device calls for advanced numerical models. One of the simplest numerical methods is the vortex panel method [13] which 45 offers some understanding of the turbine's hydrodynamics but has severe limitations such as assuming that lift and drag coefficients are constant over one revolution or the omission of the effects of unsteadiness and turbulence.

Computational Fluid Dynamics (CFD) has become a powerful tool for predictions of the operation and performance of tidal turbines. Tidal turbine CFD simulations are generally based on the Reynolds Averaged Navier-Stokes (RANS) equations as they are less demanding in terms of computational cost than other CFDapproaches. Maitre et al. [14] used a 2D RANS $k$ - $\omega$ SST model to reproduce the experimental results from Roa et al. [8] and they found that this approach tends to overestimate the experimental results which was related 55 to the lack of consideration of $3 \mathrm{D}$ effects in the model. McNaughton et al. [15] reproduced the setup from [14] adding the Low-Reynolds number Effect (LRE) correction to the 2D RANS $k$ - $\omega$ SST model and improved results were obtained with the change of the turbulence model although they still were unable to match well the experimental data. Similar analysis was done by Howell et al. [16] using ${ }_{60} 2 \mathrm{D}$ and $3 \mathrm{D}$ RANS with the $k-\varepsilon$ RNG turbulence model for a vertical axis wind 
turbine (VAWT). The lack of accuracy of the 2D RANS model was shown again, highlighted by a large overestimation of the power coefficient, whilst using the 3D model reduced the overestimation significantly demonstrating the importance of reproducing the 3D nature of the flow around vertical axis turbines. ${ }_{65}$ Marsh et al. [17] analysed the influence of different helicolidal configurations on a VATT using 3D RANS which provided relevant outcomes to understand the impact of this geometrical variation onto the device's performance.

One reason for the lack of accuracy of RANS based approaches to predict flow-turbine interaction is that flow-turbine interactions are governed by largescale turbulence. The method of LES is, in theory, more suited to simulate these interactions due its demonstrated higher accuracy compared to RANS in predicting flows dominated by large-scale energetic vortices. However, the computational requirements of LES are much higher than those of RANS and this is a limitation to most researchers and practitioners [18, 19, 20]. Most LES 75 studies are in the area of vertical axis wind turbines (VAWTs): Iida et al. [21] compared results of momentum theory-based calculations with LES achieving a good match between them although no comparison with experimental data was performed. Li et al. [22] performed a more complete analysis comparing outputs of 2D and 2.5D RANS and LES with performance data of a VAWT [9].

80 They demonstrated the higher accuracy of LES compared to RANS but point out that the vertical extension of the domain may have an appreciable impact on the final results. Elkhoury et al. [23] performed the LES of a full 3D VAWT model including the analysis of the effects of blade shape and fixed or variable pitch angle on the VAWT's performance. Their LES results agreed well with experimental data. Furthermore, complementary LES and experiments demonstrated that the power coefficient of the VAWT can be increased by employing variable-pitch turbine blades. Recently, Posa et al. [24] performed a LES of a VATT using the immersed boundary method. The numerical results were well validated with experimental data in terms of wake velocity and turbulent 90 kinetic energy profiles, although they do not report the performance predicted with LES and compare it with the experimental data. 
In this paper, a large-eddy simulation approach is refined with a tailormade Immersed Boundary (IB) method with the goal to allow accurate bladeresolved simulations of a vertical axis tidal turbine. The methodology couples 95 dynamically a Lagrangian-based solid turbine to the Eulerian-based fluid and avoids expensive computational processes that arise when using blade-resolved methods, e.g. variable reallocation and/or interpolation at every time step [25]. The IB method was introduced by Peskin [26] for the simulation of heart valves. Since then, the IB method has been used in a wide range of applications such as particle laden flows [27], fluid-structure interaction [28], bluff bodies in depthaveraged shallow water models [29] or simulation of HATTs [30], and has proven to work well for flows with moving boundaries.

The objective of this study reported here is to demonstrate the applicability and accuracy of the chosen IB method-based LES to simulate a VATT and to provide insights into the complex blade-vortex interactions of the VATT in operation. The paper is organised as follows: in Section 2 the method is introduced with special emphasis on the chosen immersed boundary method in Section 2.2. Then the model is thoroughly validated for a VATT subjected to a 2D laminar approach flow in Section 3. Section 4 shows an in-depth study of 110 a VATT subjected to turbulent flow: error convergence of the LES-IB method, hydrodynamic coefficients and power performance compared to the results from RANS-based approaches and experiments. The visualisation of the blade-vortex interaction at different tip speed ratios and the power spectral density within the turbine's swept area are presented in Section 5 with the goal to shed light on the complex flow-turbine interaction. The paper finishes with conclusions drawn from the outcomes of this study in Section 6 .

\section{Numerical method}

\subsection{Navier-Stokes equations solver}

The well-validated in-house code Hydro3D ([31, 32, 33, 34, 35, 36, 37, 38, 39]) is used in the current research. The flow is solved using the spatially filtered 
Navier-Stokes equations for turbulent, incompressible, three-dimensional flow which read,

$$
\begin{aligned}
& \nabla \cdot \mathbf{u}=0 \\
& \frac{\partial \mathbf{u}}{\partial t}+\mathbf{u} \cdot \nabla \mathbf{u}=-\nabla p+\frac{1}{R e} \nabla^{2} \mathbf{u}-\nabla \cdot \tau+\boldsymbol{f}
\end{aligned}
$$

where $\mathbf{u}=(\mathrm{u}, \mathrm{v}, \mathrm{w})^{T}$ and $p$ are the fluid velocities and pressure, $\tau$ is the subgrid scale stress tensor, $R e$ is the Reynolds number based on the chord length ( $R e=$ $c U_{0} / \nu$, where $U_{0}$ is the inlet velocity, $c$ is the hydrofoil's chordlength, and $\nu$ is the fluid kinematic viscosity), and $\boldsymbol{f}=\left(f_{x}, f_{y}, f_{z}\right)^{T}$ is the volume force from the IB method [27].

In Hydro3D the Eulerian fluid flow is solved through finite differences with staggered storage of the velocity components on a Cartesian grid. Fourth-order central differences with a combination of a third-order low-storage Runge-Kutta method for convective and diffusive terms respectively are used in the prediction step of the fractional-step method $[40,41]$. The solution of a Poisson pressurecorrection equation is achieved using the multi-grid technique in the final step 130 as a corrector of the predicted velocities. The subgrid scale stresses are approximated by the Wall-Adapting Local Eddy-viscosity (WALE) [42] subgrid scale (sgs) model. WALE is particularly suitable when used in combination with the immersed boundary method because it does not require explicit treatment of the sgs-viscosity near solid boundaries [43]. In order to represent the moving solid boundaries of the VATT in an Eulerian fluid domain, the direct forcing IB method [27] is used and detailed in-depth in the Section 2.2. Hydro3D runs on massively parallel high performance computers and features a recently developed and validated local mesh refinement approach [43] that minimises the computational cost of LES. The solution domain is decomposed via domain decomposition into a certain number of sub-domains and their communication is accomplished using the Message Passing Interface (MPI) protocol.

The fractional-step method based on a three-step Runge-Kutta pressure- 
correction is formulated as follows:

$$
\begin{aligned}
& \frac{\tilde{\mathbf{u}}-\mathbf{u}^{l-1}}{\Delta t}=\frac{\alpha_{l}}{R e} \nabla^{2} \mathbf{u}^{l-1}-\alpha_{l} \nabla p^{l-1}-\alpha_{l}[\mathbf{u}(\nabla \cdot \mathbf{u})]^{l-1}-\beta_{l}[\mathbf{u}(\nabla \cdot \mathbf{u})]^{l-2} \\
& \tilde{\mathbf{u}}^{*}=\tilde{\mathbf{u}}+\boldsymbol{f} \Delta t \\
& \nabla^{2} \tilde{p}=\frac{\nabla \cdot \tilde{\mathbf{u}}^{*}}{\alpha_{l} \Delta t} \\
& \mathbf{u}^{t}=\tilde{\mathbf{u}}^{*}-\alpha_{l} \Delta t \nabla \tilde{p} \\
& p^{t}=p^{t-1}+\tilde{p}-\frac{\alpha_{l} \Delta t}{2 R e} \nabla^{2} \tilde{p}
\end{aligned}
$$

Here $l=1,2,3$ denotes the Runge-Kutta sub-step for which $l=1$ denote values from the previous time step $t$ - 1 and the Runge-Kutta coefficients are $\alpha_{1}=\beta_{1}=1 / 3$, $\alpha_{2}=\beta_{2}=1 / 6$ and $\alpha_{3}=\beta_{3}=1 / 2$. $\tilde{\mathbf{u}}$ and $\tilde{\mathbf{u}}^{*}$ are the nón-solenoidal predicted Eulerian velocities before and after the immersed boundary method correction respectively, $\mathbf{u}^{t-1}$ and $\mathbf{u}^{t}$ are the Eulerian velocity at the previous and present time steps, $\tilde{p}$ is the pseudo-pressure, $p$ is the pressure, and $\boldsymbol{f}$ is the source term due to the IB method.

\subsection{The immersed boundary method}

There are two main types of IB approaches (and many variations of both [44]) depending on how the immersed body is represented. The first type represents the desired body shape as a continuous boundary, e.g. as in [45], while the second type discretises the body shape through a set of regularly spaced Lagrangian markers, e.g. [27]. The discrete approach appears to deal well with moving bodies which is why the IB method presented here builds on the discrete IB approach known as the direct forcing method introduced by Fadlun et al. [46] and later improved by Uhlmann [27]. The direct forcing method utilises solid (Lagrangian) markers that exert a force $\boldsymbol{f}$ on surrounding (Eulerian) fluid nodes (or cells) with the goal to correct the Eulerian velocity and to enforce a desired velocity at the marker's location. Fig. 1 shows the staggered arrangement of the velocities and pressure cells together with the two-dimensional representation of the closest neighbours for a Lagrangian marker (represented by a solid red circle) when using the $\phi$ kernel function from Roma et al. [47]. 
Figure 1: Two-dimensional representation of the Cartesian grid neighbours used in the interpolation for a Lagrangian marker. $\mathbf{x}$ represents pressure nodes, $\square$ are $x$-velocity nodes, $\bigcirc$ are $y$-velocity nodes, and red circles are the Lagrangian markers. $\Delta V_{L}$ denotes the Lagrangian marker volume.

The multi-step procedure of the direct forcing IB method is adapted as

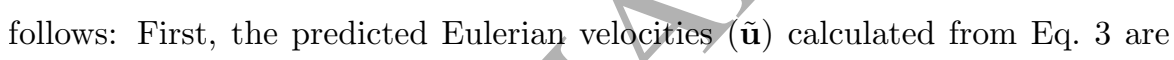
interpolated to the Lagrangian grid using delta functions $(\delta)$, which transfer the information from Lagrangian marker $L$ to its closest $n_{e}$ Eulerian neighbours. The three-dimensional delta function is calculated from one-dimensional kernel functions as:

$$
\delta\left(\mathbf{x}_{i j k}-\mathbf{X}_{L}\right)=\frac{1}{\Delta \mathbf{x}_{i j k}} \phi\left(\frac{x_{i j k}-X_{L}}{\Delta x}\right) \phi\left(\frac{y_{i j k}-Y_{L}}{\Delta y}\right) \phi\left(\frac{z_{i j k}-Z_{L}}{\Delta z}\right)
$$

Here $\mathbf{x}_{i j k}$ is the location of the Eulerian cell $i j k, \mathbf{X}_{L}$ is the location of the Lagrangian marker $L, \Delta \mathbf{x}_{i j k}=\Delta x \cdot \Delta y \cdot \Delta z$ is the Eulerian cell volume, and $\phi$ is the one-dimensional kernel function from [47]. The interpolated velocity at the Lagrangian points $\left(\mathbf{U}_{L}\right)$ is calculated from a selected number of neighbour Eulerian nodes, $n_{e}$, determined by the kernel function width, as:

$$
\mathbf{U}_{L}=\sum_{i j k=1}^{n_{e}} \tilde{\mathbf{u}}_{i j k} \cdot \delta\left(\mathbf{x}_{i j k}-\mathbf{X}_{L}\right) \cdot \Delta \mathbf{x}_{i j k}
$$

The force the Lagrangian marker $L$ needs to exert onto the fluid $\left(\mathbf{F}_{L}\right)$ in order to satisfy the no-slip condition on the immersed boundary is calculated as the difference between the desired (or forced) velocity at the marker $\left(\mathbf{U}_{L}^{*}\right.$, explained 
in Section 2.3), and the previously interpolated velocity from the fluid $\mathbf{U}_{L}[27]$ as:

$$
\mathbf{F}_{L}=\frac{\mathbf{U}_{L}^{*}-\mathbf{U}_{L}}{\Delta t}
$$

In the backwards step, the closest $n_{L}$ Lagrangian markers to each Eulerian cell transfer back the force $\mathbf{F}_{L}$ to obtain the Eulerian force $\boldsymbol{f}$ as:

$$
\boldsymbol{f}\left(\mathbf{x}_{i j k}\right)=\sum_{L=1}^{n_{L}} \mathbf{F}_{L} \cdot \delta\left(\mathbf{X}_{L}-\mathbf{x}_{i j k}\right) \cdot \Delta V_{L}
$$

The volume assigned to each Lagrangian marker $\Delta V_{L}$ is calculated as the total area/volume of the submerged body divided by the total number of Lagrangian markers [48]. The value of $\Delta V_{L}$ is approximately equal to that of $\Delta \mathbf{x}_{i j k}$ in order to ensure the forces interpolated during the forwards and backwards step are equal, i.e. $\sum \boldsymbol{f}\left(\mathbf{x}_{i j k}\right) \Delta x_{i j k}=\sum \mathbf{F}_{L} \Delta V_{L}$. In the final step the predicted Eulerian velocity $(\tilde{\mathbf{u}})$ is updated in Eq. 4 to obtain the corrected Eulerian velocity after the IB method correction $\left(\tilde{\mathbf{u}}^{*}\right)$.

\subsection{Application of the IB method to vertical axis tidal turbines}

For the present application of the IB method to vertical axis tidal turbines, the turbine blade geometry is discretised into a set of Lagrangian markers. A front-tracking algorithm [49] is developed to generate the desired hydrofoil shape, e.g. a NACA airfoil profile, as an unstructured mesh, and also provides geometrical properties such as plan area or centre of gravity. Fig. 2 shows the main geometrical parameters used for the description of the blade's circular movement, e.g. reference axis and angles. The blades rotate at a prescribed constant rotational velocity, $\Omega$, about the turbine's central shaft denoted by $\mathbf{C}_{\mathbf{x}}$ that reads,

$$
\mathbf{C}_{\mathbf{x}}=\left(C_{x}, C_{y}, C_{z}\right)^{T}
$$

For the time advancement of the blades a fixed time step is employed, which avoids spurious oscillations on the immersed boundary forces triggered when using a variable time step, as reported in [50]. The turbine rotates about the 


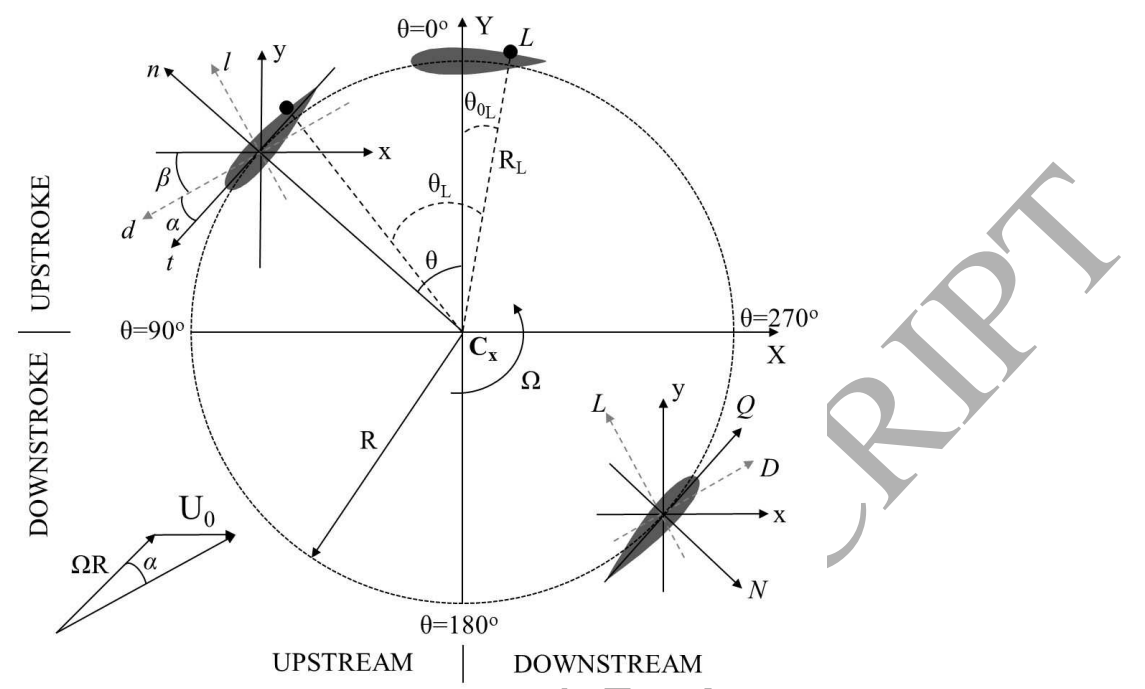

Figure 2: Graphical description of the main parameters to be considered for the rotational movement of the turbine.

Z-axis (into the paper) as depicted in Fig. 2 and for the calculation of velocities and coordinates the rotation matrix $\mathbb{R}_{z}$ is employed:

$$
\mathbb{R}_{z}\left(\theta_{L}\right)=\left(\begin{array}{ccc}
\cos \left(\theta_{L}\right) & \sin \left(\theta_{L}\right) & 0 \\
-\sin \left(\theta_{L}\right) & \cos \left(\theta_{L}\right) & 0 \\
0 & 0 & 1
\end{array}\right)
$$

Here $\theta_{L}$ stands for the rotated angle by the Lagrangian marker $L$. At the initial position of the turbine, i.e. $\theta=0^{\circ}$, the local Cartesian coordinates $\left(\mathbf{X}_{L_{0}}\right)$, the initial rotated angle $\left(\theta_{L_{0}}\right)$, and the radius $\left(R_{L}\right)$ of each Lagrangian marker comprising the three turbine blades are calculated, as:

$$
\begin{aligned}
& \mathbf{X}_{L_{0}}=\left(X_{L_{0}}, Y_{L_{0}}, Z_{L_{0}}\right)^{T} \\
& R_{0}=\sqrt{\left(X_{L_{0}}\right)^{2}+\left(Y_{L_{0}}\right)^{2}} \\
& \theta_{L_{0}}=\operatorname{atan}\left(Y_{L_{0}} / X_{L_{0}}\right)
\end{aligned}
$$

The rotated angle at time $t$ is $\theta_{L}(t)=\theta_{0_{L}}+\Omega t$ and hence the position of the 
Lagrangian markers $\left(\mathbf{X}_{L}\right)$ at a given time step can be calculated as:

$$
\begin{aligned}
& \mathbf{X}_{L}=\left(X_{L}, Y_{L}, Z_{L}\right)^{T} \\
& \mathbf{X}_{L}=\mathbf{C}_{\mathbf{x}}+\mathbb{R}_{z}\left(\theta_{L}\right) \times \mathbf{X}_{L_{0}}
\end{aligned}
$$

The direct forcing IB method algorithm requires the forced boundary velocity $\left(\mathbf{U}_{\mathbf{L}}^{*}\right)$ to calculate the reaction force $\mathbf{F}_{L}$ from Eq. 10, and is computed as the temporal rate of change of the marker position as:

$$
\mathbf{U}_{\mathbf{L}}^{*}=\left(U_{L}^{*}, V_{L}^{*}, W_{L}^{*}\right)^{T}=\frac{\partial \mathbf{X}_{L}}{\partial t}=\left(\frac{\partial X_{L}}{\partial t}, \frac{\partial Y_{L}}{\partial t}, \frac{\partial Z_{L}}{\partial t}\right)^{T}
$$

However, due to the rotational motion of the blades, which are describing a circular movement, the use of polar coordinates alternatively to the Cartesian coordinates eases the notation of the variables representing the VATT. Hence, Eq. 20 is employed to compute the Lagrangian marker coordinates in polar coordinates while Eq. 21 is used to compute the forcedLagrangian marker velocity.

$$
\begin{aligned}
& \mathbf{X}_{L}=\left(\begin{array}{c}
X_{L} \\
Y_{L} \\
Z_{L}
\end{array}\right)=\left(\begin{array}{c}
C_{x} \\
C_{y} \\
C_{z}
\end{array}\right)+\left(\begin{array}{c}
-R_{L} \cdot \sin \left(\theta_{L}\right) \\
R_{L} \cdot \cos \left(\theta_{L}\right) \\
Z_{L_{0}}
\end{array}\right) \\
& \mathbf{U}_{\mathbf{L}}^{*}=\left(\begin{array}{c}
U_{L}^{*} \\
V_{L}^{*} \\
W_{L}^{*}
\end{array}\right)=\left(\begin{array}{c}
-R_{L} \cdot \Omega \cdot \cos \left(\theta_{L}\right) \\
-R_{L} \cdot \Omega \cdot \sin \left(\theta_{L}\right) \\
0
\end{array}\right)
\end{aligned}
$$

Fig. 2 sketches the blades of the VATT together with the two coordinate systems used, all relevant geometrical variables as well as acting fluid forces (lift $L$ and $\operatorname{drag} D$ ) and the normal $n$ and tangential $t$ vectors to the blade movement used to determine the normal $N$ and torque $Q$ forces respectively. The solid reaction force $\left(\mathbf{F}=\left(F_{x}, F_{y}, F_{z}\right)\right)$ acting on each blade is determined as the sum of the forces from Eq. 10 of all $N_{L}$ markers comprising each blade as:

$$
\mathbf{F}=\int_{\text {blade }} \rho \mathbf{F}_{L} d V_{L}=\sum_{L=1}^{N_{L}} \rho \mathbf{F}_{L} \Delta V_{L}
$$

where $\rho$ is the fluid density. The turbines presented here are considered infinitely long (i.e. assuming geometrical two-dimensionality of the system) and hence 
forces in the vertical are irrelevant and are not reported. The local angle of attack, $\alpha$, is calculated according to [9] as:

$$
\alpha=\operatorname{atan}\left(\frac{-\sin (\theta)}{\lambda+\cos (\theta)}\right)
$$

where $\lambda\left(=\Omega R / U_{0}\right)$ is known as the tip speed ratio. The effective angle of attack, $\beta$, is computed as:

$$
\beta=\theta-\alpha
$$

Drag and lift forces on the blades are calculated using the $\mathrm{x}$ - and $\mathrm{y}$ - components of $\mathbf{F}$ projected on the reference frame $(l, d)$ using the angle $\beta$ as follows:

$$
\left(\begin{array}{l}
D \\
L
\end{array}\right)=\left(\begin{array}{cc}
\cos (\beta) & \sin (\beta) \\
-\sin (\beta) & \cos (\beta)
\end{array}\right)\left(\begin{array}{c}
F_{x} \\
F_{y}
\end{array}\right)
$$

The drag $\left(C_{D}\right)$ and lift $\left(C_{L}\right)$ coefficients are normalised by the tangential rotational velocity $\Omega R$ as (e.g. [14], [15]):

$$
\begin{aligned}
C_{L} & =\frac{L}{1 / 2 \rho(\Omega R)^{2} c H} \\
C_{D} & =\frac{D}{1 / 2 \rho(\Omega R)^{2} c H}
\end{aligned}
$$

The torque and normal forces are then obtained by projecting the lift and drag over the local angle of attack, $\alpha$, representing the effective parallel and perpendicular directions according to the effective local velocity. Eq. 28 shows how the torque and normal forces are calculated and their coefficients (Eq. 29 and 30) are normalised by the free-stream velocity $\left(U_{0}\right)$ instead of the tangential speed.

$$
\begin{gathered}
\left(\begin{array}{l}
Q \\
N
\end{array}\right)=\left(\begin{array}{cc}
\cos (\alpha) & \sin (\alpha) \\
-\sin (\alpha) & \cos (\alpha)
\end{array}\right)\left(\begin{array}{c}
D \\
L
\end{array}\right) \\
C_{Q}=\frac{Q}{1 / 2 \rho U_{0}^{2} A} \\
C_{N}=\frac{N}{1 / 2 \rho U_{0}^{2} A}
\end{gathered}
$$

Here $A=2 R H$ is the turbine's projected area. The resultant power coefficient, $C_{P}$, is calculated as the ratio between the power generated by the turbine $\left(P_{T}\right)$, 
i.e. summing the individual contribution from the number of blades, $N_{b}$, that comprises the device, and the available in the water $\left(P_{W}\right)$ as:

$$
C_{P}=\frac{P_{T}}{P_{W}}=\frac{N_{b} \cdot \Omega \cdot Q \cdot R}{1 / 2 \rho U_{0}^{3} A}
$$

\section{VATT subjected to laminar flow}

The first validation test of the IB-based LES approach is a three-bladed Darrieus turbine subjected to laminar flow. This VATT has been simulated previously with sophisticated body-fitted CFD models by Ferrer et al. [51] and Ramirez et al. [25] who employed a high-accuracy Galerkin method and a highorder finite volumes method, respectively, and their results are used for model comparison. The chosen test case allows quantitative assessment of the accuracy of the predictions of normal and tangential forces and the method's ability to resolve the fluid-structure interaction which generates the torque at the turbine shaft. Further, the absence of the effécts of turbulence eliminates uncertainties regarding the accurate representation of laminar-to-turbulent boundary layer transition or the impact of large- and small-scale turbulence.

The setup and boundary conditions of a 3-bladed VATT driven by laminar flow are chosen analogue to the ones of Ferrer et al.[51] and Ramirez et al. [25]. All dimensions are normalised by the chord length of the turbine blade, $c$. The radius of the VATT is $R=2 c$, the prescribed rotational velocity is $\Omega=0.5 \mathrm{rad} / \mathrm{s}$ and the inlet velocity is set to $U_{0}=0.5$. The resulting chord-based Reynolds number $\left(R e_{c}\right)$ is 100 and the tip speed ratio is 2 . The numerical domain extends $24 c$ in the streamwise and $18 c$ in the spanwise direction. The turbine centre is placed at $9 c$ from the inlet and in the middle of the channel with regards to the spanwise direction. The mesh resolution is similar to the two reference cases and is approximately $\Delta x=\Delta y=0.02 c$. For the simulations a fixed time-step is employed which avoids artificial force peaks [50]. Three different time step values are used, i.e. $\mathrm{CFL}=0.8,0.45$ and $0.2\left(\right.$ where $\left.\mathrm{CFL}=\lambda \cdot U_{0} \cdot \Delta t / \Delta x\right)$, with the goal to assess the effect of time step value on the accuracy of the simulation. 
Figure 3: Streamwise velocity contours at $\theta=720^{\circ}$ for the VATT under laminar flow.

Fig. 3 presents streamwise velocity contours at the instant in time when the turbine rotates $\theta=720^{\circ}$. The velocity contours near the blades are smooth and are comparable with the ones présented in Ramirez et al. [25]. Noteworthy are the distinct features of VATTs, which are the formation of high-velocity wakes behind the blades and the blade-wake interaction of the following blade as well as the significantly varying near-field hydrodynamics around the turbine. Regarding the latter, for instance a blade experiences low flow velocities on the upstroke side $\left(270^{\circ}<\theta<90^{\circ}\right)$ and significantly higher velocities on the downstroke side $\left(90^{\circ}<\theta<270^{\circ}\right)$.

A quantitative validation of the simulation outputs is obtained through Fig. 4 which shows the normal and torque coefficients for $360^{\circ} \leq \theta \leq 1080^{\circ}$. A remarkably good agreement of the coefficients predicted by the present IB-based simulation with the ones from the body-fitted models is achieved. There is some sensitivity of the results to the time step value wherein the smallest time step size gives the best agreement of predictions with the data from the reference calculations. In terms of the torque coefficient predictions there appears to be a better agreement with Ramirez et al. [25] on the upstream side of the cycle while the present results match better with Ferrer et al. [51] on the downstream 
Figure 4: Hydrodynamic coefficients comparison of the VATT under a laminar flow between the present and blade resolved models from [51, 25]. (a) Torque coefficient; (b) Normal coefficient.

side. Regarding the normal coefficient, the solution with the smallest timestep shows a good agreement with the referenced data both in maximum and minimum values as well as in the general distribution. Overall, the IB-based method reproduces well the flow-turbine interactions as well as the resulting 220 hydrodynamic coefficients.

\section{4.) VATT subjected to turbulent flow}

A more complex test case is the simulation of a VATT comprised of three cambered NACA 0018 blades subjected to a turbulent approach flow. This challenging test case is chosen because experimental data of Roa et al. [8] and numer-

225 ical results of Maitre et al. [14] and McNaughton et al. [15] are available to assess 
the accuracy of the present method. Both Maitre et al. [14] and McNaughton et al. [15] employed body-fitted meshes and their calculations were performed with two-dimensional Reynolds Averaged Navier-Stokes (RANS) models with $k-\omega$ SST turbulence closures. The present VATT simulations are carried out in a quasi-3D domain, i.e. using a finite 3D domain without considering turbine end-effects.

The geometrical dimensions of the test case are similar to [14] with a domain size of $30 c \times 22 c$ in $\mathrm{x}$ - and $\mathrm{y}$-directions respectively. The turbine centre is placed $9 c$ away from the inlet and in the middle of the domain regarding the lateral direction. The computational domain is extruded in the vertical by $H=2 c$, which is expected to provide accurate results. Posa et al. [24] demonstrated in their VATT simulations that using a vertical extension of $2 c$ and $4 c$ provided similar results. The radius of the turbine is $R=2.73 c$ so the resulting turbine's solidity $\left(\sigma=N_{b} c /(2 \pi R)\right)$ is 0.175 , and the Reynolds number based on the chord length is $R e_{c}=73,600$. An uniform velocity distribution of $U_{0}=2.3 \mathrm{~m} / \mathrm{s}$ is imposed at the inlet and a convective outlet condition is set at the domain's outlet. Periodic boundary conditions are set at the top and bottom boundaries while noslip conditions are imposed on the domain sides representing the experimental hydraulic flume walls. VATT simulations are performed for $\lambda=1.0,1.5,2.0,2.5$, 245 and 3.0, to obtain the performance curve and to compare in detail the present LES with the experimental results [8] and other $2 \mathrm{D}$ numerical data $[14,15]$. The fluid domain is divided into 504 sub-domains as depicted in Fig. 5 where the sub-domain divisions pattern is drawn. Three levels of local mesh refinement are used and the simulations run on the HPC Wales supercomputer using 171 Intel Xeon E5-2670 (Sandy Bridge) at 2.60GHz processors with 8 cores per socket. Each node has a total of 16 cores and 64GB RAM, and are interconnected with an Infiniband 4x QDR/PCIe gen2 16x network infrastructure (40Gbps HS/LL QDR, 1.2ms latency). The simulations on the finest mesh require approximately 24,000 CPU hours. The simulations are run initially for a period of two turbine revolutions to develop fully the flow around the turbine and power coefficients are computed and averaged over four additional revolutions. 
Figure 5: Sketch of the domain used for the current LES with the sub-domain divisions represented by solid lines.

\subsection{Grid size and time step sensitivity}

A grid size and time step sensitivity study is carried out for the turbine rotating at $\lambda=2.0$. Table 1 lists the details of the grid spacing normalised by the chord length, the number of solid markers distributed over each section of the blades' boundary, $N_{L}$, and the predicted power coefficient. These values refer to the horizontal plane where the mesh is uniform in $\mathrm{x}$ - and $\mathrm{y}$-directions, i.e. $\Delta x=\Delta y$, while the resolution in the vertical direction is set as $\Delta z=2 \Delta x$. The effect of the time step on the prediction of $C_{P}$ is quantified using the finer mesh with constant time steps of $\Delta t^{*}=\Delta t U_{0} / c=7.2 \cdot 10^{-4}, 4.0 \cdot 10^{-4}, 1.0 \cdot 10^{-4}$, corresponding to $\mathrm{CFL}=0.046,0.026,0.006$, respectively, where $\mathrm{CFL}=U_{t i p} \Delta t / \Delta x$, for which $U_{\text {tip }}=\Omega R$ is the tangential velocity of the blade. It is found that the impact of the time step size on the coefficient of power is negligible small (less than 1\%) in comparison to the sensitivity to the mesh size.

270 Fig. 6 presents the instantaneous values of the power coefficient $\left(C_{P}\right)$ obtained for one blade over one revolution for the three different meshes using $\Delta t^{*}=4.0 \cdot 10^{-4}$. A notable variation of $C_{P}$ is observed with each refinement of the mesh, i.e. meshes $\Delta x_{1}$ and $\Delta x_{2}$ show a larger and sharp $C_{P}$ peak during the blade upstroke movement with a maximum of $C_{P} \approx 0.55$ while the finest 275 mesh $\Delta x_{3}$ achieves a maximum of $C_{P} \approx 0.48$. The latter also shows a smoother 


\begin{tabular}{|c|c|c|c|}
\hline Mesh & $\Delta x / \mathrm{c}$ & $N_{L}$ & $C_{P}$ \\
\hline$\Delta x_{1}$ & 0.025 & 82 & 0.252 \\
$\Delta x_{2}$ & 0.0125 & 162 & 0.313 \\
$\Delta x_{3}$ & 0.010 & 202 & 0.328 \\
\hline
\end{tabular}

Table 1: Details of the mesh resolutions tested, the number of divisions along the airfoil's surface, $N_{L}$, and the generated power coefficient.

curve around the maximum $C_{P}$ in comparison to the pointy peaks predicted by the coarser meshes, which can be attributed to a more accurate representation of dynamic stall by the fine grid simulation, as explained later in Section 5. Table 1 shows that with successive mesh refinement thé predicted power coefficient achieves a closer match to the experimental value of $C_{P}=0.336$ reported in Maitre et al. [14], with $\Delta x_{3}$ showing only a $2.5 \%$ error.

Figure 6: Comparison of the computed power coefficient using different mesh resolutions.

The numerical simulation of a rotating turbine blade using an IB method is a challenging task. An important measure to the stability and accuracy of any immersed boundary method is to eliminate/minimise artificial residual ve-

285 locities inside the moving immersed boundary $[52,28]$. These can be quantified with the $L_{2}$ norm error, calculated as $L_{2}=\sqrt{\sum_{L=1}^{N_{L}}\left(\mathbf{U}_{L}^{*}-\mathbf{U}_{L}\right)^{2} / N_{L}}$, where $N_{L}$ indicates the total number of Lagrangian markers comprising one blade, $\mathbf{U}_{L}$ is 
the interpolated Lagrangian velocity, and $\mathbf{U}_{L}^{*}$ is the forced Lagrangian velocity. The lower the $L_{2}$ norm error, the better is the achievement of the no-slip condition at the solid boundary [52]. Hence the residual velocities inside the blades are quantified through the $L_{2}$ norm error for different spatial resolutions using a fixed time step of $\Delta t^{*}=4.0 \cdot 10^{-4}$. Fig. 7a) plots the $L_{2}$ norm error as a function of grid spacing (solid line) together with sloped (dashed) lines indicating the order of the scheme. The data line is parallel to the 1st-order slope, and hence the method is 1st-order accurate in space. Simulations to investigate the effect of temporal resolution on the $L_{2}$ norm error are carried on the $\Delta x_{2}$ gridl. Fig. $7 \mathrm{~b}$ ) plots the $L_{2}$ norm error as a function of time step (solid line) and it demonstrates that the method is 2nd-order accurate in time. Noteworthy is the fact that for a rotating VATT the accuracy in space is only linear whereas both [27] and [52] demonstrated 2nd-order accuracy of the direct forcing method, however, those were for non-turbulent flows around fixed immersed bodies such as the TaylorGreen vortex problem. Similar error convergence rates to the present ones were found by Taira et al. [53] or Li et al. [54], who attributed this deviation to the interpolation procedures or to the effects of turbulence, respectively.

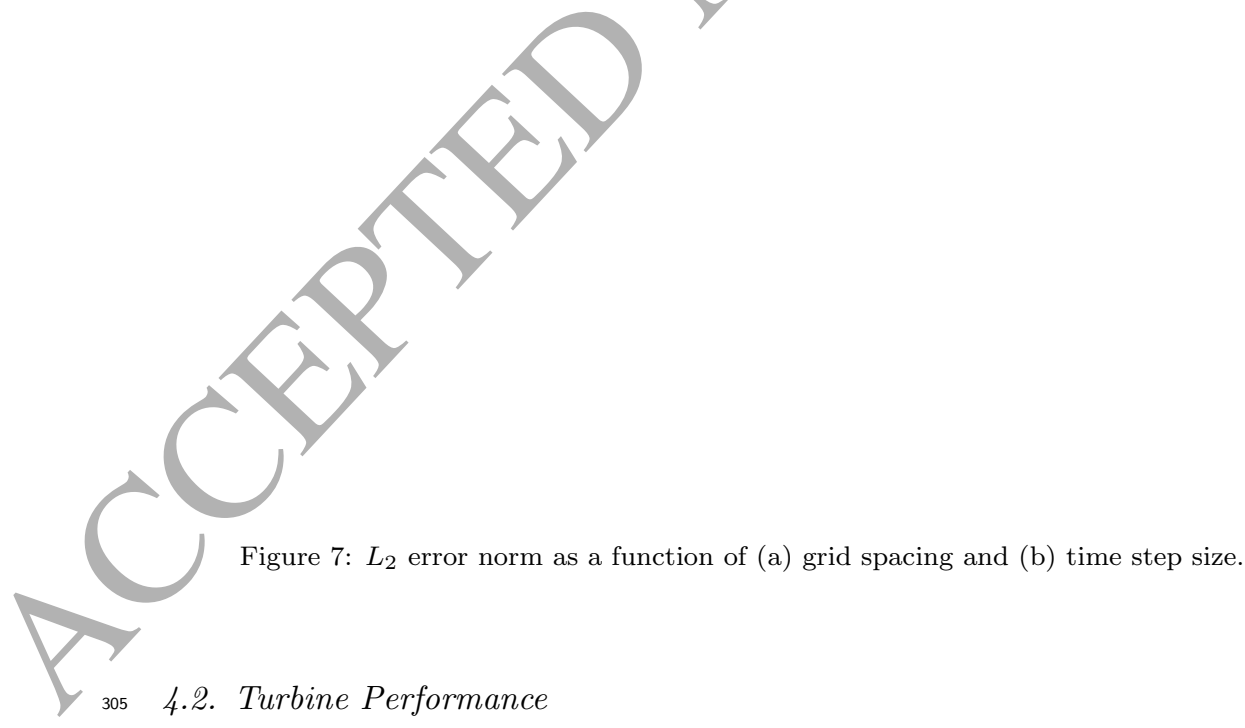

A blade resolved high-resolution numerical simulation of a VATT operating in fully turbulent conditions is able to reveal important flow-turbine physics and 
can aid in improving their design or help in understanding why some VATT designs perform better than others. A crucial step in this process is to validate the numerical approach and to assess its credibility. Thus, the present LES method [55] is first validated with data from experiments and previous numerical simulations from Maitre et al. [14] and McNaughton et al. [15].

A time-averaged coefficient of power, $C_{P}$, is first obtained from averaging $C_{P}$ over four revolutions after the turbine has initially rotated $720^{\circ}$. Fig. 8 presents the time history of the power coefficient of the first blade over six full revolutions, i.e. $2160^{\circ}$. During the first two revolutions the generated $C_{P}$ appears to be affected by the fact the flow field is not yet fully developed, whereas the power coefficient curve described over the following revolutions is very consistent.

Figure 8: Time history of the power coefficient of one blade over six revolutions.

Fig. 9 presents the power curve, i.e. mean coefficient of power as a function of tip speed ratio, obtained from the experiments of [8], the RANS simulations from $[14,15]$, and the present LES. It is observed that overall LES provides an accurate prediction of the mean power coefficient. In particular, the LES is able to predict the behaviour of the turbine during all phases of the operation, whereas RANS predictions show significant overestimation of the power coefficient for tip speed ratios greater than 2.0, i.e. in the transition and secondary effects regions.

A better understanding of power generation of a VATT is provided with the 
Figure 9: Mean power coefficient versus $\lambda$ comparison: Experimental [8], RANS [14, 15] and the present LES results.

help of Fig. 10, which presents the distribution of the phase-averaged power coefficient over one full turbine revolution for one blade (upper row) and for the sum of the three blades (lower row) at different tip speed ratios. Fig. 10 demonstrates the inherent peculiarity of a vertical axis turbine in that the power generation is very uneven over one revolution. The majority of power is generated on the upstream side of the VATT's revolution, i.e. $0^{\circ} \leq \theta \leq 180^{\circ}$, and there are phases where $C_{P}$ drops below zero or is close to zero, which means that one blade opposes the power generation of the two other blades. Hence, and as seen from the lower row of Fig. 10, the power generation of a threebladed VATT takes on a sinusoidal pattern per revolution with three peaks and three troughs. The overall distribution of $C_{P}$ of the single blade is quite similar amongst all numerical approaches irrespective of tip speed ratio, particularly on the upstream side of the turbine $\left(0^{\circ} \leq \theta \leq 180^{\circ}\right)$. Noteworthy is that the maximum $C_{P}$ value predicted by LES and the $k-\omega$ SST LRE [15] is quite similar for $\lambda \neq 1.5$ and 2.0 , while $k$ - $\omega$ SST features a notable overestimation at all rotational speeds. More pronounced differences between the numerical approaches are observed for $180^{\circ} \leq \theta \leq 270^{\circ}$, i.e. during the downstream side of the rotation, for which only LES predicts a rather significant secondary $C_{P}$ peak (with approx. $60 \%$ of the magnitude of the primary peak). This secondary peak is due to 
the lift overshoot experienced by the blade on the outer side as a result of the onset of a secondary trailing edge vortex, and this was also found for vertical axis wind turbines [9]. This particular blade-vortex interaction is visualised and discussed later in Section 5. The RANS models also predict secondary peaks, however they are smaller in magnitude than the LES predictions and occur at a later stage in the revolution, i.e. $\theta \approx 330^{\circ}$.

The power coefficient of the 3-bladed VATT is shown in Fig. 10d) to f) as 355 predicted by the two RANS models, LES and measured experimentally. The LES predictions for $\lambda=1.5$ match the experimental values both in phase and extreme values. At maximum efficiency, i.e. $\lambda=2.0$, LES slightly overestimates the maximum $C_{P}$ and similarly underestimates the troughs of the curve. The LES predicted $C_{P}$ curve also features a slight shift in the location of the peaks and troughs, possibly due a slight shift of the $\theta=0^{\circ}$ position in the experiments. At a higher tip speed ratio of $\lambda=2.5$, LES predicts well the mean value but fails in the prediction of the maxima and minima values, which is probably due to the omission of end-effects, which have a greater effect at higher rotational velocities.

The accurate prediction of the lift coefficient of individual blades by the numerical model is of great importance and essential for geometry-resolved Darrieus-type VATT simulations, because they are lift driven devices. A detailed analysis of hydrodynamic lift forces is enabled by Fig. 11, which presents the lift coefficient, $C_{\mathcal{L}}$, as a function of angle of attack, $\alpha$, for tip speed ratios of 370 $\lambda=1.5,2.0$ and 2.5. Significant differences between LES and RANS are found along the $C_{L}$ curve irrespective of the rotational speed. The most relevant differences concern the lift generated during the first half of the blade's upstroke movement $\left(0<\alpha<\alpha_{\max }\right.$ and $\left.0^{\circ} \leq \theta \leq 90^{\circ}\right)$, where LES predicts a higher generation than RANS approaches at all $\lambda$ with differences up to a $20 \%$.

Fig 11a) shows the lift hysteresis loop for $\lambda=1.5$ and suggests large differences among the different numerical approaches. The LES predicts the transition of power generation to loss of lift to occur at $\theta \approx 70^{\circ}$ while this is delayed until $\theta \approx 80^{\circ}$ in the RANS predictions. Consequently, the LES predicts that 
Figure 10: Coefficient of power, $C_{P}$, as a function of rotated angle, $\theta$, of one turbine blade for $\lambda=1.5$ (a), 2.0 (b) and 2.5 (c), and $C_{P}$ of the entire turbine for $\lambda=1.5$ (d), 2.0 (e) and 2.5 (f). Experimental and RANS $k-\omega$ SST data from [14], RANS $k-\omega$ SST LRE from [15], and present LES.

at $\theta=90^{\circ}$ (blade is perpendicular to the oncoming flow) $C_{L} \approx 1.8$ whereas the

380 RANS models predict $C_{L} \approx 2.8$, reflecting the differences between approaches in predicting the energetic flow structures that dominate the flow over the blade. Nonetheless, at the maximum effective angle of attack $\left(\alpha=40^{\circ}, \theta=130^{\circ}\right)$ the three approaches converge to the same value of $C_{L}$. At this angle, the blade suffers from detachment of the dominating large-scale structure (as shown later in Section 5) causing the lift to drop dramatically. The considerable differences between numerical approaches are due to their different prediction of dynamic stall that dominates the VATT physics at low tip speed ratios, $\lambda<1.7$ [14]. In the last quarter of rotation $\left(270^{\circ} \leq \theta \leq 360^{\circ}\right)$, the generated lift is negative as 
the large-scale vortices are formed on the blade's outer surface, and where LES predicts less generation of lift in comparison to RANS models.

Figure 11: Coefficient of lift, $C_{L}$, as a function of effective angle of attack, $\alpha$, of one turbine blade for $\lambda=1.5$ (a), $\lambda=2.0$ (b) and $\lambda=2.5$ (c). Comparison of the present LES results with RANS results $[14,15]$.

The hysteresis lift curves for $\lambda=2.0$ and 2.5 are presented in Fig. 11b) and c), respectively. The difference in the predictions of lift during $\alpha>0$, where LES consistently exceeds the magnitude of $C_{L}$ compared to that of RANS, exists for almost the entire upstream rotation for both rotational speeds. However, the peak of $C_{L}$ is predicted to occur at $\theta \approx 90^{\circ}$ for $\lambda=2.0$ and 2.5 irrespective of the numerical approach. Larger differences are again observed for the downstream side of the reyolution especially for $270^{\circ} \leq \theta \leq 360^{\circ}$. The source of the differences is the resolution of the blade-vortex interaction as RANS approaches tend to underestimate the formation of leading and trailing edge vortices which are the principal factors affecting the generation of lift on the blades.

Fig. 12 presents the drag coefficient curves for tip speed ratios of 1.5, 2.0 and 2.5. At $\lambda=1.5$, two regions of high $C_{D}$ values are predicted by LES and the peaks are located at $\theta \approx 90^{\circ}$, i.e. coinciding with maximum power generation, and at $\theta \approx 210^{\circ}$ in the third quarter of the rotation. The first LES-predicted peak 405 is about $30 \%$ larger than the maximum values of $C_{D}$ from RANS simulations. 
These differences are due to a better resolution of the dynamic stall on the blades (as explained later in Section 5) by the LES, which becomes more evident in the prediction of the secondary peak. For $\lambda=2.0$ and 2.5, predicted magnitudes of the coefficient of drag are similar among the numerical approaches, however the secondary peak predicted by LES is located between $180^{\circ} \leq \theta \leq 270^{\circ}$ whereas RANS models estimate it to occur at $270^{\circ} \leq \theta \leq 360^{\circ}$. This is consistent with the findings for the $C_{P}$ curves of Fig. 10a) to c).

igure 12: Coefficient of drag, $C_{D}$, as a function of rotated angle, $\theta$, of one turbine blade for $\lambda=1.5$ (a), $\lambda=2.0$ (b) and $\lambda=2.5$ (c). Comparison of the present LES results with RANS results $[14,15]$.

\section{Blade-yortex interaction}

An inherent fluid-structure interaction phenomenon of Darrieus-type VATTs is dynamic stall. This is characterised by the shedding of leading and trailing edge vortices from the blade in motion, which is more pronounced on the upstream side of the circumference that the blade describes. These vortices are consequently convected through the swept area of the rotor and hence they may interact again with the blade during its rotation on the downstream half 420 of the circumference. The complex vortex shedding and blade-vortex interaction is sketched in Fig. 13, as proposed by Brochier et al. [56] who investigated experimentally dynamic stall characteristics of a three-bladed VATT rotating 
at $\lambda=2.14$. From their observations, various vortex shedding mechanisms take place, mainly in the form of leading and trailing edge vortices, denoted as $\boldsymbol{a}$ and $\boldsymbol{b}$ respectively. Fig. 13 illustrates their loci of generation, growth and pathways of travel through the rotor's swept area.

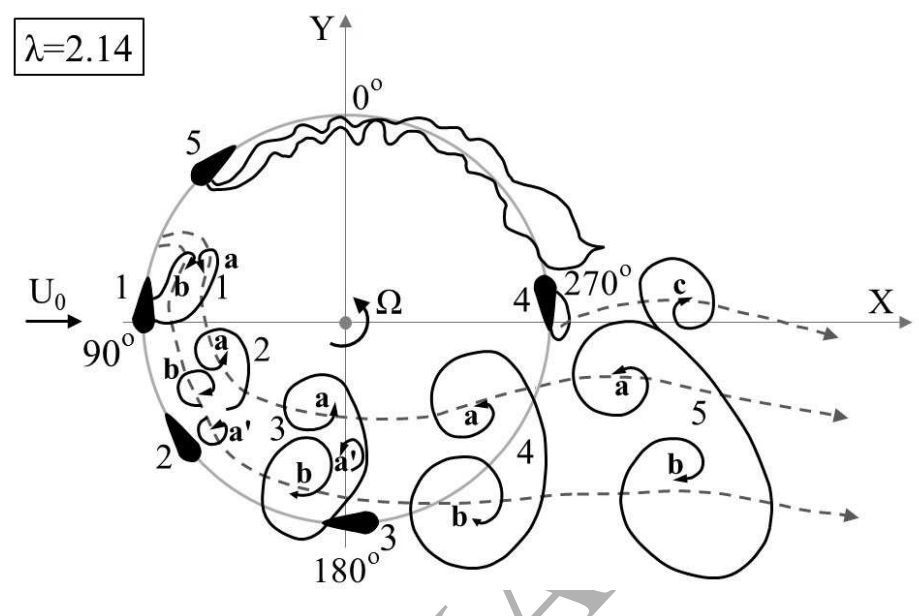

Figure 13: Schematic of the vortex shedding sequence of a VATT at $\lambda=2.14$ described by Brochier et al. [56].

Dynamic stall characteristics depend on the tip speed ratio the turbine is operating at. Fig. 14 shows the effective angle of attack $\alpha$ (Eq. 23) of the blade during its upstream rotation at different tip speed ratios. The static and dynamic stall angles, denoted as $\alpha_{s s}$ and $\alpha_{d s}$ respectively, determine whether the blade experiences light or deep dynamic stall, and hence whether partial or full flow separation takes place and at which angle it is occurring. The lower the tip speed ratio, the larger is the region the blade operates under deep stall conditions (see Fig. 14b)) and this results in premature flow separation. At 435 higher rotational speeds, the blade motion is dominated by inertial effects and the flow separation on the inner side of the blade is notably reduced. In the latter, $\alpha$ is less than $\alpha_{d s}$ so light stall occurs instead of deep stall.

The ability of LES to resolve the large-scale structures in the instantaneous velocity field together with a geometry-resolved approach permits studying in 440 detail dynamic stall and vortex-blade interactions of a VATT, which vary no- 
Figure 14: a) Effective angle of attack $(\alpha)$ developed by the VATT blade during the first half of the revolution for different tip speed ratios, where $\alpha_{s s}$ and $\alpha_{d s}$ are the stall angles under static and dynamic airfoil motion conditions. b) The shaded grey areas indicate where the VATT blade undergoes deep stall when rotating at $\lambda=1.5$.

tably depending on the tip speed ratio. Three different scenarios are analysed: $\lambda=1.0,2.0$ and 3.0. At $\lambda=1.0$ and 1.5 deep dynamic stall occurs and the turbine stops operating or experiences a severe drop in performance, respectively. At higher tip speed ratios, e.g. $\lambda=2.5$ and 3.0 , the inertia of the blades dom445 inates over viscous effects and the chosen turbine "overspins", in other words it does not operate at its peak. For the turbine under investigation the peak performance is at $\lambda \approx 2.0$, see Fig. 9 , which appears to be the best compromise between maximum lift on the upstream portion of the revolution and minimum negative lift on the downstream portion of the revolution.

Fig. 15 visualises coherent vortices through iso-surfaces of the vertical vorticity for $\lambda=2.0$ illustrating the blade-vortex interaction and vortex evolution at 9 different rotated angles. The iso-surfaces are coloured in blue and orange indicating whether their rotation is clockwise and counter-clockwise, respectively. At position $\mathrm{I}\left(\theta \approx 30^{\circ}\right)$, the blade does not experience any vortex shedding and only minor trailing edge turbulence is observed. Once the blade passes the dynamic stall angle, i.e. at position II, onset of dynamic stall occurs with the generation of an energetic Leading Edge Vortex (LEV) denoted $\boldsymbol{a}$ and at the instant depicted it already occupies most of the blade's suction side. The blade generates the maximum torque at $\theta \approx 90^{\circ}$ (see Fig. 10b) despite or probably 
because of the LEV $\boldsymbol{a}$. At position III, vortex $\boldsymbol{a}$ is considerably larger but has been already advected away from the suction side of the blade and is replaced by a Trailing Edge Vortex (TEV) $\boldsymbol{b}$, which is rotating in the opposite direction to the LEV. The TEV is growing as the blade continues its motion and smaller LEVs are generated such as vortex $\boldsymbol{a}^{\prime}$. At position IV, the primary LEV $\boldsymbol{a}$ is fully detached from the blade and is now being advected through the inside of the rotor whereas the TEV $\boldsymbol{b}$ grows and dominates the blade's hydrodynamics. The TEV $\boldsymbol{b}$ is eventually shed from the blade at $\theta \approx 180^{\circ}$, as is observed at position $\mathrm{V}$. At the tip speed ratio of 2.0, vortices shed during the upstream side of the motion interact with the blades mostly within the third quarter of the rotation, i.e. $180^{\circ}<\theta<270^{\circ}$. This is visible at position $\mathrm{V}, \theta \approx 230^{\circ}$, where the blade interacts with the advected TEV from the previous blade, whilst its own TEV $\boldsymbol{b}$ has detached completely. At position VI the blade starts to generate a secondary LEV $\boldsymbol{c}$ on the blade's outside and the blade now interacts with the LEV of the previous blade $\boldsymbol{a}$. Once the blade reaches position VII, no more blade vortex interaction takes place and only small leading and trailing edge vortices form, detach and are transported into the downstream area of the turbine. In the fourth quarter, there is no influence of upstream turbulence and the blades at positions VHI and IX experience only vortex shedding at the trailing edge and in absence of any relevant flow separation. The dotted lines indicate the path described by the centre of the vortices $\boldsymbol{a}$ and $\boldsymbol{b}$, and all of the findings and observations agree remarkably well with Brochier et al.'s [56] sketch.

Significant dynamic stall is observed when the turbine is rotating at $\lambda=1.0$ and this is appreciated with help of Fig. 16. At position I, only laminar shear layers on both sides of the blades are visible. The rapid onset of dynamic stall, 485 massive flow separation in the form of a LEV $\boldsymbol{a}$ is appreciated already at position II and a TEV $\boldsymbol{b}$ is starting to form as well. Both vortices, $\boldsymbol{a}$ and $\boldsymbol{b}$, are being shed even before the blade approaches $\theta \approx 90^{\circ}$. From flow visualisations it is observed that this vortex shedding mechanism does not always follow the same pattern, sometimes vortex $\boldsymbol{b}$ is shed, some other times it stays attached to the 
Figure 15: Blade-vortex interaction of the VATT at $\lambda=2.0$.

and motion affects the shedding of the TEV. A secondary LEV $\boldsymbol{a}^{\prime}$ is generated at position III, and is shed before the blade advances to position IV whilst the secondary TEV $\boldsymbol{b}$ ' remains attached. Note that at a tip speed ratio of 1.0 at an angle of $\theta=180^{\circ}$ the relative velocity between the blade and free-stream is zero. This provokes that at position $\mathrm{V}$ the vortex $\boldsymbol{b}^{\prime}$ travels close to the blade at a similar velocity but is physically detached. The vortex $\boldsymbol{b}^{\prime}$ quickly loses coherence due to its interaction with vortex $\boldsymbol{a}^{\prime}$ which rotates in the opposite direction. The same is true for the main vortices $\boldsymbol{a}$ and $\boldsymbol{b}$, they interact with each other on their way through the turbine and for the scenario depicted here the TEV has lost its coherence almost entirely by the time it exits the rotor on the downstream side.

Significant $\boldsymbol{a}$ " and TEV $\boldsymbol{b}$ " are being generated and shed at position VI. At position VII, the blade experiences interaction with vortices $\boldsymbol{a}$ and $\boldsymbol{b}$, shed 
Figure 16: Blade-vortex interaction of the VATT at $\lambda=1.0$.

during the first quarter of the revolution and another trailing edge vortex ap-

pears $(\boldsymbol{a} "$ "). The interaction continues until position VIII, and appear to affect the shedding of $a$ " . Leading edge flow separation is still observed at position VIII and also clearly at position IX and the full recovery of the boundary layer on the outer side of the blade is not achieved until the blade passes $\theta=0^{\circ}$.

Fig. 17 visualises blade-vortex-interactions when the turbine rotates at $\lambda=3.0$, and this is when the turbine overspins and operates below its peak efficiency. The turbine does not undergo deep dynamic stall at positions I and II at which only minor trailing edge vortices form and the shear layers on both sides are still attached to the blade. The LEV $\boldsymbol{a}$ is generated at position III and shed shortly afterwards because of the fast motion of the blade. At position IV the blade 515 is under light stall with a short region of flow separation closer to the trailing edge, which generates small-scale flow structures. This behaviour is expected as Fig. 14a) shows that $\alpha$ is never larger than $\alpha_{d s}$ for $\lambda=3.0$. Note that light 


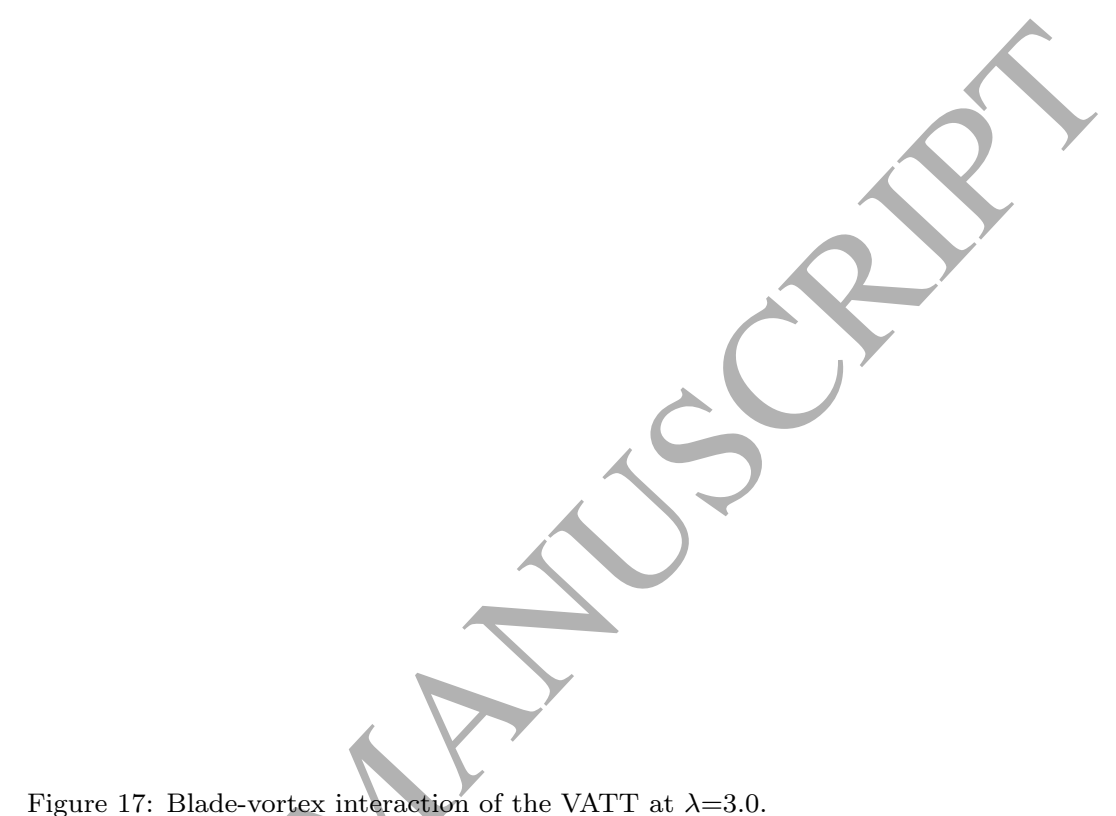

Figure 17: Blade-vortex interaction of the VATT at $\lambda=3.0$.

or deep stall conditions are differentiated by the extension of the separated flow region, extending only over a short portion of the blade's surface or over the entire length of the blade, respectively.

The region of flow separation region near the inner side of the blade is already reduced at position $\mathrm{V}$ and gone entirely by position VI. In contrast, there is no flow separation at the outside of the blade at position $\mathrm{V}$, while at VI a secondary LEV $\boldsymbol{a}^{\prime}{ }^{\prime}$ is generated and is already located close to the trailing edge. The vortex $\boldsymbol{a}$ (shed at the position III) interacts with the following blade somewhere between position V and VI. At position VII, vortex $\boldsymbol{a}$ " is already detached and advected downstream of the turbine. Minor but not insignificant vortex formation and shedding is observed for the remainder of the revolution and until position I.

${ }_{530}$ Visualised by Fig. 15 to 17 the blade-vortex interaction depends strongly on the tip speed ratio $\lambda$. Noteworthy is the difference in the pathways described 
by the vortices shed on the upstream side of the revolution. For $\lambda=1.0$, the vortices travel in the direction of the main flow as the low rotational velocity allows for a larger entrainment of fluid into the turbine's swept area. The swept area is more isolated from the approach flow in the cases of $\lambda=2.0$ and 3.0 and consequently the vortices are deflected to the right, e.g. in the case of $\lambda=3.0$ by approx. $45^{\circ}$.

\subsection{Power spectral density}

Signals of instantaneous $u$ - and $v$-velocity are collected at a point located at $\mathrm{x} / \mathrm{R}=0.80$ and $\mathrm{y} / \mathrm{R}=-0.60$, and denoted as " $\mathrm{P}$ " in Fig. 15 for the case of the turbine rotating at $\lambda=2.0$. This point is placed within the pathway of the LEV $\boldsymbol{a}$ vortices that are shed during dynamic stall around $\theta=90^{\circ}$. The angular velocity of the turbine is $\Omega=52.57 \mathrm{rad} / \mathrm{s}$, which results in a blade frequency of $f_{b}=(\Omega / 2 \pi)^{*} N_{b}=25.1 \mathrm{~s}^{-1}$, or blade period of $T_{b}=0.04 \mathrm{~s}$ which is used to normalise the time values of the velocity signals from Fig. 18a). The Power Spectral Density (PSD) from the velocity signals is presented in Fig. 18b). A large energy peak associated with the passing blade is identified at $f_{b}$ in both PSDs together with another distinct peak at a lower frequency of $f_{r}=3.8 \mathrm{~s}^{-1}$. The latter is the frequency of the circular flow motion inside the turbine swept area induced by the rotation of the turbine's rotor, which was also observed by Brochier et al. [56] from transversal velocity spectra. A secondary, highfrequency peak is also present in the spectra and this is due to the fact that previously shed yortices are convected through the rotor's swept area and hence are out of sync with the blade movement, showing up as additional energetic 555 spikes in the spectra.

The low-frequency peak of the circular fluid motion and the blade-vortex PSD peaks are found in the production range of the spectrum. As Fig. 18b) shows the energy cascade of the $u$ and $v$ spectra exhibit a certain range of isotropic turbulence decay following the expected -5/3 decay and from approximately $10^{3} \mathrm{~Hz}$ onwards energy dissipation takes place, mainly induced by the SGS model. The selected mesh resolves approx. three frequency decades of the 

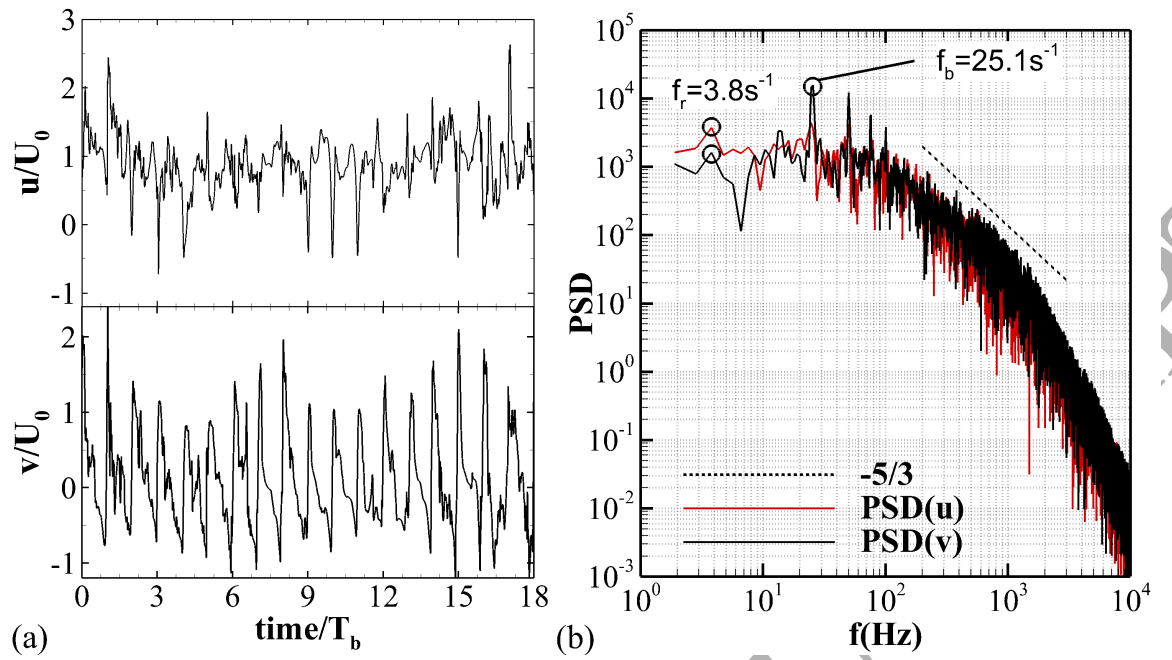

Figure 18: a) Time series of $u$ - and $v$-velocities and their b) Power Spectral Density (PSD) at $\mathrm{x} / \mathrm{R}=0.80, \mathrm{y} / \mathrm{R}=-0.60$.

flow, between the production of energetic large-scale vortices and dissipation of small-scale turbulence, further demonstrating the adequacy of the chosen mesh [57].

\section{Conclusions}

In this paper an immersed boundary-based large-eddy simulation for the simulation of Darrieus-type vertical axis tidal turbines has been introduced, validated and applied. In the first instance, a 3-bladed turbine subjected to laminar flow has been simulated and results have been compared with two highly-accurate body-fitted methods. Predicted torque and normal coefficients are generally in good agreement with the outputs of the two methods used for comparison, and this has demonstrated the stability and accuracy of the large-eddy simulation based immersed boundary (LES-IB) method in absence of turbulence.

575 Then the LES-IB method has undergone detailed performance assessment by comparing simulation results with experimental and RANS-based model data 
for a 3-bladed VATT subjected to turbulent flow. A mesh resolution and time step sensibility study has been carried out first to assess optimal grid and time step sizes for this case. The turbine performance curve has been reproduced with the predicted power predictions, which agree very well with the ones obtained from experiments. Further, it demonstrates that the LES-IB method has outperformed the two RANS-based models, in particular when the turbine spins at tip speed ratios above the optimum tip speed ratio. The detailed analysis of generated power and acting lift forces over a single revolution has revealed significant differences in terms of when power/lift is generated during the rotation and this analysis has highlighted that important blade-vortex interaction takes place. In particular, the largest differences between LES and RANS predictions in the lift coefficient have been found at a tip speed ratio of 1.5, which is when dynamic stall dominates the VATT's hydrodynamics and RANS model appear to struggle to reproduce this phenomenon accurately.

The visualisation of the blade-vortex interaction at three different tip speed ratios has outlined that the generation and transport of energetic large-scale flow structures depends strongly on the rotational speed of the turbine. At low to moderate rotational speeds, the blades undergo deep dynamic stall, i.e. massive flow separation from the leading edge of the blade and formation of leading edge vortices that are approximately the size of the blade chord length. On the contrary, at high rotational velocities the blades experience light dynamic stall and vortices are less significant. The analysis of power density spectra together with the visualisations have confirmed the presence of deep stall vortices. The spectra allowed quantification of their dominating frequency and have provided evidence of the complex blade-vortex interaction mechanisms.

\section{Acknowledgements}

This research was founded by the UK Engineering and Physical Science Research Council (EPSRC), grant no. EP/K502819/1. This work was performed using the computational facilities of the Advanced Research Computing 
@ Cardiff (ARCCA) Division, Cardiff University and HPC Wales. The authors would like to thank Dr. Luis Ramirez and Dr. Xesús Nogueira (Universidade da Coruña) and Dr. Esteban Ferrer (Universidad Politécnica de Madrid) for providing their numerical results.

\section{References}

[1] R. Martin-Short, J. Hill, S. Kramer, A. Avdis, P. Allison, M. Piggott, Tidal resource extraction in the Pentland Firth, UK: Potential impacts on flow regime and sediment transport in the Inner Sound of Stroma, Renew. Energy 76 (2015) 596-607. doi:10.1016/j.renene.2014.11.079.

[2] M. Sánchez, R. Carballo, V. Ramos, G. Iglesiás, Tidal stream energy impact on the transient and residual flow in an estuary: A 3D analysis, Appl. Energy 116 (2014) 167-177. doi:10.1016/j.apenergy .2013.11.052.

[3] L. Chen, W. H. Lam, A review of suryivability and remedial actions of tidal current turbines, Renew. Sustain. Energy Rev. 43 (2015) 891-900. doi:10.1016/j.rser.2014.11.071.

[4] A. Copping, L. Hanna, J. Whiting, S. Geerlofs, M. Grear, K. Blake, A. Coffey, M. Massua, J. Brown-Saracino, H. Battey, Environmental effects of marine energy development around the world annex IV final report, Tech. Rep. January (2013). doi:10.1016/j.ocecoaman.2014.04.002.

${ }_{625}$ [5] E. P. R. Institute, Environmental Effects of Hydrokinetic Turbines on Fish, Tech. rep., Electric Power Research Institute (2012).

[6] M. Khan, G. Bhuyan, M. Iqbal, J. Quaicoe, Hydrokinetic energy conversion systems and assessment of horizontal and vertical axis turbines for river and tidal applications: A technology status review, Appl. Energy 86 (10) (2009) 1823-1835. doi:10.1016/j. apenergy .2009.02.017. 
[7] S. Kiho, M. Shiono, K. Suzuki, The power generation from tidal currents by darrieus turbine, Renew. Energy 9 (1-4) (1996) 1242-1245. doi:10. 1016/0960-1481 (96) 88501-6.

[8] A. M. Roa, V. Aumelas, T. Maître, C. Pellone, Numerical and experimental 635 analysis of a darrieus-type cross flow water turbine in bare and shrouded configurations, in: 25th IAHR Symp. Hydraul. Mach. Syst., Vol. 12, IOP Publishing, 2010, p. 012113. doi:10.1088/1755-1315/12/1/012113.

[9] K. McLaren, Unsteady loading of high solidity vertical axis wind/ turbines, Phd thesis, McMaster University (2011).

${ }_{640}[10]$ A. J. Fiedler, S. Tullis, Blade Offset and Pitch Effects on a High Solidity Vertical Axis Wind Turbine, Wind Eng. 33 (3) (2009) 237-246. doi:10. $1260 / 030952409789140955$.

[11] L. Priegue, T. Stoesser, The influence of blade roughness on the performance of a vertical axis tidal turbine, Int. J. Mar. Energy 17 (2017) 136146. doi:10.1016/j.ijome.2017.01.009.

[12] P. Bachant, M. Wosnik, Performance measurements of cylindrical- and spherical-helical cross-flow marine hydrokinetic turbines, with estimates of exergy efficiency, Renew. Energy 74 (2015) 318-325. doi:10.1016/j . renene.2014.07.049.

${ }_{650}^{60}$ [13] M. Islam, D. S. K. Ting, A. Fartaj, Aerodynamic models for Darrieus-type straight-bladed vertical axis wind turbines, Renew. Sustain. Energy Rev. 12 (4) (2008) 1087-1109. doi:10.1016/j.rser.2006.10.023.

[14] T. Maître, E. Amet, C. Pellone, Modeling of the flow in a Darrieus water turbine: Wall grid refinement analysis and comparison with experiments, Renew. Energy 51 (2013) 497-512. doi :10.1016/j.renene.2012.09.030.

[15] J. McNaughton, F. Billard, A. Revell, Turbulence modelling of low Reynolds number flow effects around a vertical axis turbine at a range 
of tip-speed ratios, J. Fluids Struct. 47 (2014) 124-138. doi:10.1016/j. jfluidstructs.2013.12.014.

${ }_{660}^{6}$ [16] R. Howell, N. Qin, J. Edwards, N. Durrani, Wind tunnel and numerical study of a small vertical axis wind turbine, Renew. Energy 35 (2) (2010) 412-422. doi:10.1016/j.renene.2009.07.025.

[17] P. Marsh, D. Ranmuthugala, I. Penesis, G. Thomas, Numerical investigation of the influence of blade helicity on the performance characteristics of vertical axis tidal turbines, Renew. Energy 81 (2015)]926-935. doi:10.1016/j.renene.2015.03.083.

[18] W. Rodi, G. Constantinescu, T. Stoesser, Large-Eddy Simulation in Hydraulics, IAHR Monographs, CRC Press, Leiden, The Netherlands, 2013.

[19] T. Stoesser, Large-eddy simulation in hydraulics: Quo Vadis?, J. Hydraul. Res. 52 (4) (2014) 441-452. doi:10.1080/00221686.2014.944227.

[20] M. Kear, B. Evans, R. Ellis, S. Rolland, Computational Aerodynamic Optimisation of Vertical Axis Wind Turbine Blades, Appl. Math. Model.doi: 10.1016/j.apm.2015.07.001.

[21] A. Iida, K. Kato, A. Mizuno, Numerical simulation of unsteady flow and aerodynamic performance of vertical axis wind turbines with LES, in: 16th Australas. Fluid Mech. Conf., Australia, 2007, pp. 1295-1298.

[22] C. Li, S. Zhu, Y.-1. Xu, Y. Xiao, 2.5D large eddy simulation of vertical axis wind turbine in consideration of high angle of attack flow, Renew. Energy 51 (2013) 317-330. doi:10.1016/j.renene.2012.09.011.

[23] M. Elkhoury, T. Kiwata, E. Aoun, Experimental and numerical investigation of a three-dimensional vertical-axis wind turbine with variable-pitch, J. Wind Eng. Ind. Aerodyn. 139 (2015) 111-123. doi:10.1016/j·jweia. 2015.01 .004 . 
[24] A. Posa, C. M. Parker, M. C. Leftwich, E. Balaras, Wake structure of a single vertical axis wind turbine, Int. J. Heat Fluid Flow (2016) 1-10doi: 10.1016/j.ijheatfluidflow.2016.02.002.

[25] L. Ramírez, C. Foulquié, X. Nogueira, S. Khelladi, J.-C. Chassaing, I. Colominas, New high-resolution-preserving sliding mesh techniques for higher-order finite volume schemes, Comput. Fluids 118 (2015) 114-130. doi:10.1016/j.compfluid.2015.06.008.

[26] C. Peskin, Flow patterns around heart valves: A numerical method, J. Comput. Phys. 10 (2) (1972) 252-271. doi:10.1016/0021-9991(72) 90065-4.

[27] M. Uhlmann, An immersed boundary method with direct forcing for the simulation of particulate flows, J. Comput. Phys. 209 (2) (2005) 448-476. doi:10.1016/j.jcp.2005.03.017.

[28] M. C. Kara, T. Stoesser, R. McSherry, Calculation of fluidstructure interaction: methods, refinements, applications, Proc. ICE - Eng. Comput. Mech. 168 (2) (2015) 59-78. doi:10.1680/eacm.15.00010.

700 [29] P. Ouro, L. Cea, L. Ramírez, X. Nogueira, An immersed boundary method for unstructured meshes in depth averaged shallow water models, Int. J. Numer. Methods Fluids 81 (11) (2016) 672-688. doi:10.1002/fld.4201.

[30] P. Ouro, M. Harrold, T. Stoesser, P. Bromley, Hydrodynamic loadings on a horizontal axis tidal turbine prototype, Submitt. to J. Fluids Struct.

705 [31] T. Stoesser, V. I. Nikora, Flow structure over square bars at intermediate submergence: Large Eddy Simulation study of bar spacing effect, Acta Geophys. 56 (3) (2008) 876-893. doi:10.2478/s11600-008-0030-1.

[32] T. Stoesser, Physically Realistic Roughness Closure Scheme to Simulate Turbulent Channel Flow over Rough Beds within the Framework of LES, J. Hydraul. Eng. 136 (October) (2010) 812-819. doi:10.1061/(ASCE) HY. 1943-7900.0000236. 
[33] S. Bomminayuni, T. Stoesser, Turbulence Statistics in an Open-Channel Flow over a Rough Bed, J. Hydraul. Eng. 137 (11) (2011) 1347-1358. doi: 10.1061/(ASCE) HY.1943-7900.0000454.

715 [34] M. C. Kara, T. Stoesser, K. Will, A numerical method to predict fluidstructure interaction of flow past an elastically mounted circular cylinder., in: Proc. 22nd Int. Offshore Polar Eng. Conf. ISOPE, Rhodes, Greece., 2012 .

[35] D. Kim, T. Stoesser, J.-H. Kim, The effect of baffle spacing on hydrodynamics and solute transport in serpentine contact tanks, J. Hydraul. Res. 51 (5) (2013) 558-568. doi:10.1080/00221686.2013.777681.

[36] S. Kara, M. C. Kara, T. Stoesser, T. W. Sturm, Free-Surface versus RigidLid LES Computations for Bridge-Abutment Flow, J. Hydraul. Eng. 141 (9) (2015) 04015019. doi:10.1061/(ASCE)HY.1943-7900.0001028.

725 [37] S. Kara, T. Stoesser, T. W. Sturm, S. Mulahasan, Flow dynamics through a submerged bridge opening with overtopping, J. Hydraul. Res. 53 (2) (2015) 186-195. doi:10.1080/00221686.2014.967821.

[38] B. Fraga, T. Stoesser, Influence of bubble size, diffuser width, and flow rate on the integra1 behavior of bubble plumes, J. Geophys. Res. Ocean. 121 (6) (2016) 3887-3904. doi:10.1002/2015JC011381.

[39] B. Fraga, T. Stoesser, C. C. Lai, S. A. Socolofsky, A LES-based EulerianLagrangian approach to predict the dynamics of bubble plumes, Ocean Model. 97 (2016) 27-36. doi:10.1016/j.ocemod.2015.11.005.

[40] A. J. Chorin, Numerical solution of the Navier-Stokes equations, Math. Comput. 22 (104) (1968) 745-762. doi:10.1090/ S0025-5718-1968-0242392-2.

[41] A. Cristallo, R. Verzicco, Combined Immersed Boundary/Large-EddySimulations of Incompressible Three Dimensional Complex Flows, Flow, Turbul. Combust. 77 (2006) 3-26. doi:10.1007/s10494-006-9034-6. 
[42] F. Nicoud, F. Ducros, Subgrid-scale stress modelling based on the square of the velocity gradient tensor, Flow, Turbul. Combust. 62 (3) (1999) 183-200. doi : 10.1023/A: 1009995426001 .

[43] M. Cevheri, R. McSherry, T. Stoesser, A local mesh refinement approach for large-eddy simulations of turbulent flows, Int. J. Numer. Methods Fluids 82 (2016) 261-285. doi:10.1002/fld.421.

[44] F. Sotiropoulos, X. Yang, Immersed boundary methods for simulating fluidstructure interaction, Prog. Aerosp. Sci. 65 (2014) 1-21. doi:10.1016/ j.paerosci.2013.09.003.

[45] S. Kang, I. Borazjani, J. A. Colby, F. Sotiropóulos, Numerical simulation of $3 \mathrm{D}$ flow past a real-life marine hydrokinetic turbine, Adv. Water Resour. 39 (2012) 33-43. doi:10.1016/j . advwatres.2011.12.012.

[46] E. Fadlun, R. Verzicco, P. Orlandi, J. Mohd-Yusof, Combined ImmersedBoundary Finite-Difference Methods for Three-Dimensional Complex Flow Simulations, J. Comput. Phys. 161 (1) (2000) 35-60. doi:10.1006/jcph. 2000.6484

[47] A. M. Roma, C. S. Peskin, M. J. Berger, An adaptive version of the immersed boundary method, J. Comput. Phys. 153 (2) (1999) 509-534. doi: $10.1006 /$ jcph.1999.6293.

[48] P. Ouro, T. Stoesser, R. McSherry, Large-Eddy Simulation of a Vertical Axis Tidal Turbine using an Immersed Boundary Method, in: CFD Wind Tidal Offshore turbines, Springer International Publishing, 2015, Ch. 5, pp. 49-58. doi:10.1007/978-3-319-16202-7_5.

[49] A. El-Hamalawi, A 2D combined advancing front-Delaunay mesh generation scheme, Finite Elem. Anal. Des. 40 (9-10) (2004) 967-989. doi: 10.1016/j.finel.2003.04.001. 
[50] S. J. Shin, W.-X. Huang, H. J. Sung, Assessment of regularized delta functions and feedback forcing schemes for an immersed boundary method, Int. J. Numer. Methods Fluids 58 (2008) 263-286. doi:10.1002/fld.1706.

[51] E. Ferrer, R. H. Willden, Bladewake interactions in cross-flow turbines, Int. J. Mar. Energy 11 (2015) 71-83. doi:10.1016/j.ijome.2015.06.001.

[52] Z. Wang, J. Fan, K. Luo, Combined multi-direct forcing and immersed boundary method for simulating flows with moving particles, Int. J. Multiph. Flow 34 (3) (2008) 283-302. doi:10.1016/j.ijmultiphaseflow. 2007.10 .004

775 [53] K. Taira, T. Colonius, The immersed boundary method: A projection approach, J. Comput. Phys. 225 (2) (2007) 2118-2137. doi:10.1016/j.jcp. 2007.03 .005

[54] R. Y. Li, C. M. Xie, W. X. Huang, C. X. Xu, An efficient immersed boundary projection method for flow over complex/moving boundaries, Comput. Fluids 140 (2016) 122-135. doi:10.1016/j.compfluid.2016.09.017.

[55] P. Ouro, T. Stoesser, Simulation of a Vertical Axis Tidal Turbine using Hydro3D - v.1.0.0, Zenodo, 2017. doi: 10.5281/zenodo. 293007.

[56] G. Brochier, P. Fraunie, C. Beguier, I. Paraschivoiu, Water channel experiments of dynamic stall on Darrieus wind turbine blades, J. Propuls. Power 2 (5) (1986) 445-449. doi : 10.2514/3.22927.

[57] J. Fröhlich, C. P. Mellen, W. Rodi, L. Temmerman, M. a. Leschziner, Highly resolved large-eddy simulation of separated flow in a channel with streamwise periodic constrictions, J. Fluid Mech. 526 (2005) 19-66. doi: 10.1017/S0022112004002812. 